\title{
Dynamical phenomena in complex networks: fundamentals and applications
}

\author{
Serhiy Yanchuk ${ }^{1, a}$, Antonio C. Roque ${ }^{2}$, Elbert E. N. Macau ${ }^{3,4}$, and Jürgen Kurths ${ }^{5,6}$ \\ 1 Institute of Mathematics, Technische Universität Berlin, Berlin, Germany \\ 2 Department of Physics, School of Philosophy, Sciences and Letters of Ribeirão Preto, University of São Paulo, Ribeirão \\ Preto, SP 14040-901, Brazil \\ 3 Federal University of São Paulo, São José dos Campos, SP, Brazil \\ 4 National Institute for Space Research, São José dos Campos, SP, Brazil \\ 5 Earth System Analysis and Complexity Science, Potsdam Institute for Climate Impact Research (PIK), Member of the \\ Leibniz Association, 14473 Potsdam, Germany \\ 6 Institute of Information Technology, Mathematics and Mechanics, Lobachevsky University of Nizhny Novgorod, Nizhny \\ Novgorod 603950, Russia
}

Published online 29 September 2021

(C) The Author(s) 2021

\begin{abstract}
This special issue presents a series of 33 contributions in the area of dynamical networks and their applications. Part of the contributions is devoted to theoretical and methodological aspects of dynamical networks, such as collective dynamics of excitable systems, spreading processes, coarsening, synchronization, delayed interactions, and others. A particular focus is placed on applications to neuroscience and Earth science, especially functional climate networks. Among the highlights, various methods for dealing with noise and stochastic processes in neuroscience are presented. A method for constructing weighted networks with arbitrary topologies from a single dynamical node with delayed feedback is introduced. Also, a generalization of the concept of geodesic distances, a path-integral formulation of network-based measures is developed, which provides fundamental insights into the dynamics of disease transmission. The contributions from the Earth science application field substantiate predictive power of climate networks to study challenging Earth processes and phenomena.
\end{abstract}

\section{Introduction}

During the last decade, networks with complex topology have become a very powerful approach for understanding large complex systems in various fields of applications ranging from neuroscience, via engineering to sociology, economy, and Earth sciences. Complex real-world systems are modeled as networks of interacting nodes, combining different application fields with approaches from dynamical system theory, statistical physics, time series analysis, and graph theory. Such systems may include different - from a few to hundreds of billions - numbers of participants, as well as much larger communication networks or the brain. Organization in networks can vary significantly, ranging from allto-all to hierarchical structures and sparse structures.

This special issue nucleated from the research performed in the framework of the eponymous GermanBrazilian International Research Training Group (IRTG) funded by the German Science Foundation DFG and the São Paulo Research Foundation FAPESP. This multidisciplinary IRTG, launched in 2011, consisted of physicists, mathematicians, climatologists,

\footnotetext{
a e-mail: yanchuk@math.tu-berlin.de (corresponding author)
}

biologists and geographers from the German and Brazilian teams, including University of São Paulo, National Institute for Space Research, Humboldt University at Berlin, Potsdam Institute for Climate Impact Research, and other institutions.

We subdivide the contributions into three main fields: theory and methods, applications to neuroscience, and Earth science applications. The theoretical aspects considered here cover a broad spectrum of topics such as collective dynamics of excitable systems, cluster dynamics [1], interplay of noise and feedback [2], subdiffusive behavior [5], spreading phenomena on networks [8], coarsening [9], multipartite networks, partial synchrony [11], and time-delayed interactions [13,14]. In a series of works, complex networks are successfully employed as tools for detecting artificially inserted data [3], community detection [4,12], identification of time series patterns [6], and modeling a voter dynamics [15].

An application focus of this special issue lies in Earth sciences and neuroscience. Among the neuroscienceoriented contributions, we present some reviews: on modeling of neural networks [16], models of intracellular calcium spikes [17], and stochastic modeling approach to networks of integrate-and-fire neurons [18]. In addition, aspects of noise modeling in spiking neuronal mod- 
els [20] and effects of hyperpolarization-activated current kinetics [19] are investigated.

A substantial part of this special issue is also devoted to Earth sciences. Special emphasis has been placed on functional climate networks $[21,26,28]$. Also, here it is shown how complex climate networks can be used as a tool to study various processes and phenomena, such as equatorial wave interactions associated with the Madden-Julian oscillation [23], dominant global covariability patterns between sea surface temperatures and precipitation [24], fires in tropical forests over space and time [25], Amazon rainfalls [27], South American low-level circulation [29], climate-induced hysteresis in pan-tropical forests [32], and others.

Below we provide a slightly more detailed overview of the contributions within this special issue.

\section{Theory and methods}

Here we mention the methodologically oriented contributions. Our classification is for convenience only and is not strict since many of the papers mentioned here are closely related to applications.

Ronge and Zaks [1] study the emergence and stability of periodic collective oscillations in systems of identical excitable units with repulsive all-to-all interaction. Special attention is put on splay states and two-cluster states.

Kromer [2] investigates the interplay of noise and an event-triggered feedback in excitable systems. Besides a review of recent results on the dynamics of a single active rotator with positive feedback, the article presents new findings on how the interplay of noise, feedback, and input from other active rotators can lead to asynchronous bursting of individual elements and collective bursting of the entire network.

Prado et al. [3] show how artificially inserted data (data that does not belong to an original stationary signal) may be detected using recurrence microstate statistics. Examples of their analyses are shown for numerically generated time series of dynamical systems and experimental time series of a human speech.

Gouvêa et al. [4] review the use of force-directed algorithms as a tool to solve the community detection problem. In particular, they highlight the most appropriate force-directed models for community detection.

Silva et al. [5] investigate the presence of sub-diffusive behavior in the Chirikov-Taylor standard map. They show that the evolution of trajectories under stickiness influence in this system can indeed be described as a sub-diffusive process. The developed procedure is even applicable to other Hamiltonian systems and also suitable to investigate transport properties on complex networks.

Anghinoni et al. [6] present a method for the identification of time series patterns using complex networks tools. The method first transforms the time series into a network according to the correlation between the different segments of the time-series. The presence of repeated hierarchical patterns is then captured through network metrics, such as the modularity.

Lima et al. [7] review recent results on the nearoptimal stochastic control problem. In particular, they focus on the approach of a discretization of the noise values instead of the canonical time-discretization.

Ianelli and Sokolov [8] introduce a path-integral formulation of network-based measures that generalizes the concept of geodesic distance and provides fundamental insights into the dynamics of disease transmission as well as efficient numerical estimation of the infection arrival time.

Valejo et al. [9] provide a review and comparative analysis of coarsening algorithms on bipartite networks. They present illustrative examples of how problems in bipartite networks can be addressed using these coarsening algorithms.

Tomasini and Lucarini [10] contribute to the response theory of spatially extended systems. A recent approach to the problem focuses on providing a method to predict changes in one observable using a second observable as a surrogate for the actual forcing. The authors present a method for quantifying and ranking the predictive ability of observables and apply it to the Lorenz '96 climate model.

Teichmann [11] investigates partially synchronous states using phase-response functions beyond first-order approximation. Three examples are presented: first, the usage of the phase-response curve for the description of macroscopic oscillators is analyzed. The next example considers a two-group Kuramoto model. In the last example, the phase dynamics of three Stuart-Landau oscillators are extended beyond the weak coupling limit.

Verri et al. [12] present a network communitydetection technique based on properties that emerge from a nature-inspired flocking system. The proposed algorithm contains two alternating mechanisms: the control the particles alignment in higher dimensional space and an iterative process of edge removal.

Freitas et al. [13] investigate the effects of time-delays and phase lags on circular formations with symmetric clusters in a first-order mobile agent model with all-toall interaction. When the information exchange timedelay exceeds some critical value, it leads to either incoherent or alternative symmetric arrangements. Besides, they show that formations can be effectively controlled using phase lags.

Stelzer and Yanchuk [14] show how a single dynamical system with time-delayed feedback can emulate networks. This property of delay systems made them extremely useful tools for Machine Learning applications. Here the authors describe several possible setups, which allow emulating multilayer (deep) feed-forward networks as well as recurrent networks.

Vega-Oliveros et al. [15] propose a voter model to study the interplay between the dynamics of opinions and the tolerance of individuals to accept others' opinions. The proposed model incorporates bots and radical or intolerant individuals in the decision-making process. The dynamics of the system occurs in a multiplex network of interacting agents composed of two layers, 
one for the dynamics of opinions where agents choose between two possible alternatives, and the other for the tolerance dynamics, in which agents adopt one of two tolerance levels.

\section{Applications to neuroscience}

The articles on applications to neuroscience show the breadth of topics in neuroscience that can be approached from the complex dynamical networks perspective.

Shimoura et al. [16] review methods of modeling the neural networks of the brain with emphasis on network organization. After briefly reviewing how simplified "point" neurons and morphologically detailed neurons and their synapses can be modeled, they proceed to give a description of the main strategies for constructing networks of both point neurons and neurons with morphology. They illustrate the reviewed approaches with a discussion of 18 recent large-scale network models of different brain regions.

Friedhoff et al. [17] make a comprehensive review of models of intracellular calcium spikes. Intracellular calcium spikes are observed in a variety of cell types and the authors show, with a detailed analysis of experimental results, that they are inherently stochastic. The authors review recent progress on stochastic models to explain the statistics of interspike intervals and discuss their shortcomings. They end with a comparison between intracellular calcium spikes and neuronal spikes and some suggestions of how methods used to describe the stochasticity of the latter can be used to model the stochasticity of the former.

Vellmer and Lindner [18] review the stochastic approach based on the Fokker-Planck equation to model networks of so-called integrate-and-fire (IF) spiking neurons. Most examples of stochastic networks of IF neurons found in the literature assume white noise input, and the framework presented by the authors even allows a systematic and consistent extension to the study of the effects of colored noise in such networks. In the second part of the article, the authors review applications of the Fokker-Planck approach to model sequences of binary decisions in cognitive neuroscience, and introduce a novel two-dimensional model of stochastic decision making which provides a good fit to presented experimental data.

Ceballos et al. [19] study how the time constant of the hyperpolarization-activated cation current $\mathrm{I}_{\mathrm{h}}$ influences dynamic neuronal membrane potential responses, especially the time course of excitatory postsynaptic potentials (EPSPs). This is an important topic in neuroscience since $I_{h}$ with a variety of kinetic properties is abundant in neurons, and the time course of synaptic potentials is a key determinant of synaptic integration. The authors perform their study on a Hodgkin-Huxleytype model of a pyramidal neuron of the hippocampus with an $I_{h}$ conductance with either fast or slow kinetics, and characterize the different impacts of the two ver- sions of the same current on the amplitude and duration of EPSPs.

Cordeiro et al. [20] present a simple way of incorporating intrinsic noise to spiking neuronal models. They consider a fire-and-reset neuron model like the integrate-and-fire neuron with a voltage-dependent spike probability function. First, the authors show how to choose the parameters of the probability function from experimental membrane voltage time series. Second, they evaluate the reliability of the response of the neuron to constant or noisy frozen stimuli and test the model with respect to stochastic resonance. Finally, they characterize, via phase diagrams, the behavior of random networks of the stochastic neuron for different stochasticity levels.

\section{Earth science applications}

Earth science is one of the application focuses of this special issue and 12 contributions are dedicated to this topic.

Ferreira et al. [21] study functional climate networks. The patterns in functional climate networks are highly important for the study of climate dynamics since they generally represent pathways for the long-distance transportation of energy. The authors provide a systematic study of the effect of 29 time series distance functions on functional climate network construction based on global temperature data. They observe that the distance functions previously used in the literature commonly generate very similar networks while alternative ones result in rather distinct network structures and reveal different teleconnections.

Raphaldini et al. [22] study fluctuations of the geomagnetic dipole field. Using two millennial scale models based on spherical harmonic fitting of paleomagnetic data, the authors find significant interactions between the dipole and smaller scale harmonics (quadrupole and octupole) of the geomagnetic field. In [23], the same authors show an application of causality ideas in complex networks in order to infer properties of equatorial wave interactions associated with the MaddenJulian oscillation (MJO), the dominant component of the atmospheric system on intraseasonal timescales in the equatorial region.

Ekhtiari et al. [24] employ a coupled climate network analysis for characterizing dominating global covariability patterns between sea surface temperatures (SST) and precipitation (PCP) at monthly timescales. Their analysis uncovers characteristic seasonal patterns associated with both local and remote statistical linkages and demonstrates their dependence on the type of the current ENSO phase.

Cano-Crespo et al. [25] implement a complex networkbased methodology to track individual fires over space and time. Focussing on extreme, the $5 \%$ most intense fires in the tropical forests of the Brazilian Legal Amazon over the period 2002-2019, they observe that major droughts, which increase forest flammability, co-occur 
with high extreme fire years. They also show the important role of anthropogenic activities.

Wolf and Donner [26] study the characteristics of functional climate networks which have been obtained by quantifying event synchrony in terms of two different similarity measures, event synchronization and event coincidence analysis. They observe that the choice of the methodological setup and the related parameters can have a substantial impact on the network topology and the conclusions.

Ciemer et al. [27] assess the direct impact of greenhouse gas-driven warming of the tropical Atlantic ocean on the Amazon rainfall. In addition, they estimate the effect of an Atlantic meridional overturning circulation (AMOC) slowdown or collapse. They find that these two processes, weakening of the AMOC and the warming of the tropical Atlantic ocean, both caused by global warming, are likely to have competing impacts on the rainfall sum in the Amazon, and hence on the stability of the Amazon rainforest.

Kittel et al. [28] employ functional climate network analysis to distinguish the global climate responses to different phases of the El Niño-Southern Oscillation (ENSO) from those to the three largest volcanic eruptions since the mid-twentieth century as the two most prominent types of recurrent climate disruptions.

Gelbrecht et al. [29] utilize streamflow wind networks, a type of climate networks that tracks the local flow of the wind field, together with the analysis of composites of wind, precipitation, and geopotential height fields, in order to investigate the variability of the South American low-level circulation.

Gelbrecht et al. [30] propose a framework for studying predictability of the first and of the second kind for high-dimensional chaotic models. The method is applied to a newly introduced multistable climate toy model constructed by coupling the Lorenz '96 and an energy balance model. Then neural ordinary differential equations are applied to predict the future state of the system.

Kittel et al. [31] demonstrate how algorithmic approaches from viability theory can be used to get a better understanding of the state space of manageable dynamical systems.

Drüke et al. [32] investigate the climate-induced hysteresis in pan-tropical forests and the impact of fire under future climate conditions. For this, they employed the Earth system model CM2Mc, which is biophysically coupled to the fire-enabled state-of-theart dynamic global vegetation model LPJmL. The results show a climate-induced hysteresis in tropical forest and lagged responses in forest recovery after the climate has returned to its original state.

Wunderling et al. [33] introduce the PyCascades open source software package for studying interacting tipping elements. The software allows for investigating under which conditions potentially dangerous cascades can emerge between interacting dynamical systems, with a focus on tipping elements.

\section{Discussion and outlook}

We hope that this volume will contribute to discussions on advances in the field of dynamical networks and stimulate further studies. For example, the software presented in [33] can be used to study the dynamics of tipping elements. Among the applications to neuroscience, there are promising ones using statistical physics methods to deal with stochastic processes, from intracellular calcium spikes [17] and single neuron firing [20] to sequential decision-making behavior [18]. The contributions from the Earth science application field clearly demonstrate the predictive power of climate networks to study challenging Earth processes and phenomena $[21,26,28]$. Among the possible further developments, the applications of complex dynamical networks to machine learning can play an important role. For example, the presented approach for constructing weighted networks with arbitrary topology from a single dynamical node with feedback [14] may increase the synergy between the community working in dynamical systems and machine learning.

Acknowledgements This work was financed by the German Research Foundation (DFG) in the framework of IRTG 1740 and project 411803875 (S.Y.), São Paulo Research Foundation FAPESP (Grant \# 2015/50122-0).

Funding Open Access funding enabled and organized by Projekt DEAL.

Open Access This article is licensed under a Creative Commons Attribution 4.0 International License, which permits use, sharing, adaptation, distribution and reproduction in any medium or format, as long as you give appropriate credit to the original author(s) and the source, provide a link to the Creative Commons licence, and indicate if changes were made. The images or other third party material in this article are included in the article's Creative Commons licence, unless indicated otherwise in a credit line to the material. If material is not included in the article's Creative Commons licence and your intended use is not permitted by statutory regulation or exceeds the permitted use, you will need to obtain permission directly from the copyright holder. To view a copy of this licence, visit http://creativecomm ons.org/licenses/by/4.0/.

\section{References}

1. R. Ronge, M.A. Zaks, Splay states and two-cluster states in ensembles of excitable units. Eur. Phys. J. Spec. Top. (2021). https://doi.org/10.1140/epjs/ s11734-021-00173-2

2. J.A. Kromer, Noise-induced dynamics of coupled excitable systems with slow positive feedback. Eur. Phys. J. Spec. Top. (2021). https://doi.org/10.1140/ epjs/s11734-021-00171-4

3. T. de Lima Prado, E.E.N. Macau, S.R. Lopes, Detection of data corruption in stationary time series 
using recurrence microstates probabilities Eur. Phys. J. Spec. Top. (2021). https://doi.org/10.1140/epjs/ s11734-021-00169-y

4. A.M.M.M. Gouvêa, T.S. da Silva, E.E.N. Macau, M.G. Quiles, Force-directed algorithms as a tool to support community detection: a review. Eur. Phys. J. Spec. Top. (2021). https://doi.org/10.1140/epjs/ s11734-021-00167-0

5. M.S. Palmero, G.I. Díaz, I.L. Caldas, I.M. Sokolov, Sub-diffusive behavior in the standard map. Eur. Phys. J. Spec. Top. (2021). https://doi.org/10.1140/epjs/ s11734-021-00165-2

6. L. Anghinoni, D.A. Vega-Oliveros, T.C. Silva, L. Zhao, Time series pattern identification by hierarchical community detection. Eur. Phys. J. Spec. Top. (2021). https://doi.org/10.1140/epjs/s11734-021-00163-4

7. L. Lima, P. Ruffino, F. Souza, Stochastic near-optimal control: additive, multiplicative, non-Markovian and applications. Eur. Phys. J. Spec. Top. (2021). https:// doi.org/10.1140/epjs/s11734-021-00185-y

8. F. Iannelli, I.M. Sokolov, Path-integral formulation of spreading processes in complex networks. Eur. Phys. J. Spec. Top. (2021). https://doi.org/10.1140/epjs/ s11734-021-00161-6

9. A.D.B. Valejo, W. de Oliveira dos Santos, M.C. Nadl, L. Zhao, A review and comparative analysis of coarsening algorithms on bipartite networks. Eur. Phys. J. Spec. Top. (2021). https://doi.org/10.1140/epjs/ s11734-021-00159-0

10. U.M. Tomasini, V. Lucarini, Predictors and predictands of linear response in spatially extended systems. Eur. Phys. J. Spec. Top. (2021). https://doi.org/10.1140/ epjs/s11734-021-00158-1

11. E. Teichmann, Using phase dynamics to study partial synchrony: three examples. Eur. Phys. J. Spec. Top. (2021). https://doi.org/10.1140/epjs/ s11734-021-00156-3

12. F.A.N. Verri, R.A. Gueleri, Q. Zheng, J. Zhang, L. Zhao, Network community detection via iterative edge removal in a flocking-like system. Eur. Phys. J. Spec. Top. (2021). https://doi.org/10.1140/epjs/ s11734-021-00154-5

13. V.L.S. Freitas, S. Yanchuk, H.L.C. Grande, E.E.N. Macau, The effects of time-delay and phase lags on symmetric circular formations of mobile agents. Eur. Phys. J. Spec. Top. (2021). https://doi.org/10.1140/ epjs/s11734-021-00153-6

14. F. Stelzer, S. Yanchuk, Emulating complex networks with a single delay differential equation. Eur. Phys. J. Spec. Top. (2021). https://doi.org/10.1140/epjs/ s11734-021-00162-5

15. D.A. Vega-Oliveros, H.L.C. Grande, F. Iannelli, F. Vazquez, Bi-layer voter model: Modeling intolerant/tolerant positions and bots in opinion dynamics. Eur. Phys. J. Spec. Top. (2021). https://doi.org/10. 1140/epjs/s11734-021-00151-8

16. R.O. Shimoura, R.F.O. Pena, V. Lima, N.L. Kamiji, M. Girardi-Schappo, A.C. Roque, Building a model of the brain: from detailed connectivity maps to network organization. Eur. Phys. J. Spec. Top. (2021). https:// doi.org/10.1140/epjs/s11734-021-00152-7

17. V.N. Friedhoff, L. Ramlow, B. Lindner, M. Falcke, Models of stochastic $\mathrm{Ca}^{2+}$ spiking-established approaches and inspirations from models of neuronal spikes. Eur. Phys. J. Spec. Top. (2021). https://doi.org/10.1140/ epjs/s11734-021-00174-1

18. S. Vellmer, B. Lindner, Fokker-Planck approach to neural networks and to decision problems - a unique method for stochastic models in computational and cognitive neuroscience. Eur. Phys. J. Spec. Top. (2021). https://doi.org/10.1140/epjs/s11734-021-00172-3

19. C.C. Ceballos, R.F.O. Pena, A.C. Roque, Impact of the activation rate of the hyperpolarization-activated current Ih on the neuronal membrane time constant and synaptic potential duration. Eur. Phys. J. Spec. Top. (2021). https://doi.org/10.1140/epjs/ s11734-021-00176-z

20. V. Lima, R.F.O. Pena, R.O. Shimoura, N.L. Kamiji, C.C. Ceballos, F.S. Borges, G.S.V. Higa, R. De Pasquale, A.C. Roque, Modeling and characterizing stochastic neurons based on in vitro voltagedependent spike probability functions. Eur. Phys. J. Spec. Top. (2021). https://doi.org/10.1140/epjs/ s11734-021-00160-7

21. L.N. Ferreira, N.C.R. Ferreira, E.E.N. Macau, R.V. Donner, The effect of time series distance functions on functional climate networks. Eur. Phys. J. Spec. Top. (2021). https://doi.org/10.1140/epjs/ s11734-021-00274-y

22. B. Raphaldini, A.S. Teruya, C.F.M. Raupp, P.L. da Silva Dias, D.Y. Takahashi, Inference of the topology of geomagnetic field multipole interactions. Eur. Phys. J. Spec. Top. (2021). https://doi.org/10.1140/ epjs/s11734-021-00201-1

23. B. Raphaldini, A.S. Teruya, C.F.M. Raupp, P.L. da Silva Dias, Information flow between MJO-related waves: a network approach on the wave space. Eur. Phys. J. Spec. Top. (2021). https://doi.org/10.1140/ epjs/s11734-021-00170-5

24. N. Ekhtiari, C. Ciemer, C. Kirsch, R.V. Donner, Coupled network analysis revealing global monthly-scale covariability patterns between sea-surface temperatures and precipitation in dependence on the ENSO state. Eur. Phys. J. Spec. Top. (2021). https://doi.org/10. 1140/epjs/s11734-021-00168-z

25. A. Cano-Crespo, D. Traxl, K. Thonicke, Spatiotemporal patterns of extreme fires in Amazonian forests. Eur. Phys. J. Spec. Top. (2021). https://doi.org/10. 1140/epjs/s11734-021-00164-3

26. F. Wolf, R.V. Donner, Spatial organization of connectivity in functional climate networks describing event synchrony of heavy precipitation. Eur. Phys. J. Spec. Top. (2021). https://doi.org/10.1140/epjs/ s11734-021-00166-1

27. C. Ciemer, R. Winkelmann, J. Kurths, N. Boers, Impact of an AMOC weakening on the stability of the southern Amazon rainforest. Eur. Phys. J. Spec. Top. (2021). https://doi.org/10.1140/epjs/s11734-021-00186-x

28. T. Kittel, C. Ciemer, N. Lotfi, T. Peron, F. Rodrigues, J. Kurths, R.V. Donner, Evolving climate network perspectives on global surface air temperature effects of ENSO and strong volcanic eruptions. Eur. Phys. J. Spec. Top. (2021). https://doi.org/10.1140/epjs/ s11734-021-00269-9

29. M. Gelbrecht, N. Boers, J. Kurths, Variability of the low-level circulation of the South American mon- 
soon analysed with complex networks. Eur. Phys. J. Spec. Top. (2021). https://doi.org/10.1140/epjs/ s11734-021-00187-w

30. M. Gelbrecht, V. Lucarini, N. Boers, J. Kurths, Analysis of a bistable climate toy model with physics-based machine learning methods. Eur. Phys. J. Spec. Top. (2021). https://doi.org/10.1140/epjs/ s11734-021-00175-0

31. T. Kittel, F. Müller-Hansen, R. Koch, J. Heitzig, G. Deffuant, J.-D. Mathias, J. Kurths, From lakes and glades to viability algorithms: automatic classification of system states according to the topology of sustainable management. Eur. Phys. J. Spec. Top. (2021). https://doi. org/10.1140/epjs/s11734-021-00262-2
32. M. Drüke, W. von Bloh, B. Sakschewski, N. Wunderling, S. Petri, M. Cardoso, H.M.J. Barbosa, K. Thonicke, Climate-induced hysteresis of the tropical forest in a fire-enabled Earth system model. Eur. Phys. J. Spec. Top. (2021). https://doi.org/10.1140/epjs/ s11734-021-00157-2

33. N. Wunderling, J. Krönke, V. Wohlfarth, J. Kohler, J. Heitzig, A. Staal, S. Willner, R. Winkelmann, J.F. Donges, Modelling nonlinear dynamics of interacting tipping elements on complex networks: the PyCascades package. Eur. Phys. J. Spec. Top. (2021). https://doi. org/10.1140/epjs/s11734-021-00155-4 